\title{
THE EFFECT OF CONSUMERS' CONSCIOUSNESS ON BRAND PERCEPTIONS: A CROSS-CULTURAL STUDY
}

\author{
Lilly Ye, Frostburg State University, USA \\ Mousumi Bose, Fairfield University, USA \\ Lou Pelton, University of North Texas, USA
}

\begin{abstract}
Despite increasing interest in understanding consumers' self-concept, there is relatively limited research on consumer's selfconsciousness, one of the foundations of self concept (Belk, 1988; Chang 2006; Fenigstein et al., 1975). In addition, a disposition to focus attention on one's self (Buss, 1980), self-consciousness needs to be further explored with another related but different concept - gender consciousness, to provide a well rounded foundation of self concept (Gould, 1996). Gender consciousness, as defined by consumer's awareness of his/her gender identity (Gould, 1996; Gould and Stern, 1989), has long been considered important in social psychology since "one's consciousness of one's own gender will influence one's feelings about oneself" (Gould and Stern, 1989, p. 130). However, this concept has received scant attention in the marketing literature. Previous literature suggests that self-consciousness and gender consciousness may provide insights to some key issues of consumer behavior, including expression of values (Millenson, 1985), manifestation of personality traits (e.g. Dichter et al., 1989) and product choices (Gould and Stern, 1989). However, these studies have focused on either selfconsciousness or gender consciousness, Thus, this study attempts to address the shortcomings in extant literature and provide knowledge base to marketing managers.
\end{abstract}

Our study specially focuses on the impact of self-consciousness and gender consciousness on consumer's brand perceptions. One central step regarding enhancing consumer's brand perceptions is to increase consumer's brand sensitivity or consciousness. Such consciousness has been well researched in extant literature and has been widely explored as an instrument for enhancing brand attitude and behavior (Liao and Wang, 2009; Nelson and McLeod, 2005; Mussey, 1997). Furthermore, an interesting perspective with respect to consumer consciousness and brand consciousness is their differential nature with respect to different cultural societies. This study targets two different cultures: that of the U.S.A and China.

Using structural equation modeling, the study found that both self-consciousness and gender consciousness have a significant impact on consumer's brand consciousness, which in turn, positively relates to consumer's brand attitudes, brand loyalty, and willingness to pay price premium. Brands, therefore, play a very important role in portraying individualistic personalities in the Western culture. Besides, need for uniqueness partially mediates the relationship between self-consciousness /gender consciousness and brand consciousness. Contrary to expectations, the findings show some interesting differences for the Chinese consumers. First, self-consciousness has a negative impact on brand consciousness. Second, gender consciousness was not related to brand consciousness. Though both self-consciousness and gender consciousness have no positive effect on brand consciousness, our findings suggest that need for uniqueness mediates the relationships. If a brand has a unique appeal, it will create a bond between Chinese consumers and brands.

Findings for the study provide insights for both marketing scholars and practitioners. At the theoretical level, the study demonstrates that self-consciousness and gender consciousness are two related but different concepts, and they can impact consumer's brand perceptions. The study also indicates the importance of consumers' need for uniqueness, especially in the Eastern culture. Specifically, need for uniqueness plays an important role in enhancing their brand consciousness and other brand perceptions for both the U.S. and Chinese consumers. At the practical level, the study encourages brand managers to focus on consumers' consciousness, including their self consciousness, gender consciousness, and brand consciousness, and thus design effective marketing campaigns to evoke consumers' consciousness.

References available upon request. 